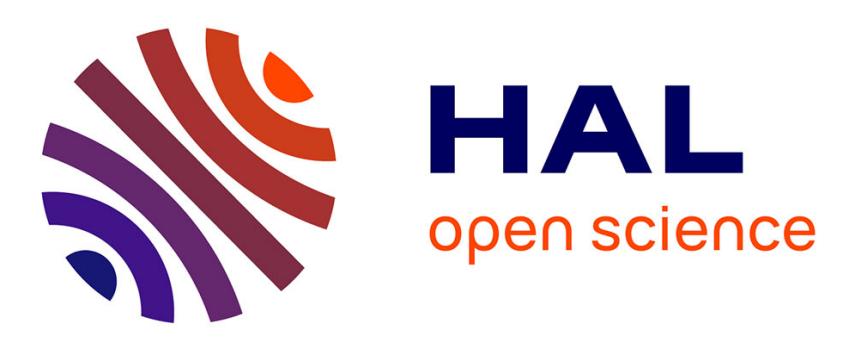

\title{
A linear algebraic approach for the computation of sums of Erlang random variables
}

Benjamin Legros, Oualid Jouini

\section{To cite this version:}

Benjamin Legros, Oualid Jouini. A linear algebraic approach for the computation of sums of Erlang random variables. Applied Mathematical Modelling, 2015, 39, pp.4971-4977. 10.1016/j.apm.2015.04.013 . hal-01265198

\section{HAL Id: hal-01265198 \\ https://hal.science/hal-01265198}

Submitted on 3 Feb 2016

HAL is a multi-disciplinary open access archive for the deposit and dissemination of scientific research documents, whether they are published or not. The documents may come from teaching and research institutions in France or abroad, or from public or private research centers.
L'archive ouverte pluridisciplinaire HAL, est destinée au dépôt et à la diffusion de documents scientifiques de niveau recherche, publiés ou non, émanant des établissements d'enseignement et de recherche français ou étrangers, des laboratoires publics ou privés. 


\title{
A Linear Algebraic Approach for the Computation of Sums of Erlang Random Variables
}

\author{
Benjamin Legros • Oualid Jouini \\ Laboratoire Génie Industriel, Ecole Centrale Paris, Grande Voie des Vignes, 92290 \\ Châtenay-Malabry, France \\ benjamin.legros@centraliens.net•oualid.jouini@ecp.fr
}

Applied Mathematical Modelling. To appear, 2015.

\begin{abstract}
We propose a matrix analysis approach to analytically provide the cumulative distribution function of the sum of independent Erlang random variables. This reduces to the characterization of the exponential of the involved generator matrix. We propose a particular basis of vectors in which we write the generator matrix. We find, in the new basis, a Jordan-Chevalley decomposition allowing to simplify the calculation of the exponential of the generator matrix. This is a simpler alternative approach to the existing ones in the literature.
\end{abstract}

Keywords: Erlang random variables, reliability, queueing systems, cumulative distribution function, Jordan-Chevalley decomposition, hypoexponential distribution.

\section{Introduction}

Many situations in service and manufacturing service systems involve the computation of the sum of independent exponential random variables. Examples include healthcare or production systems with different stages in series, system reliability with exponentially distributed components lifetimes, and wireless mobile systems with cooperative diversity schemes. This summation arises also in the transient analysis of Markovian queueing systems, and in general, semi-Markov processes.

We consider the general case of a hypoexponential distribution defined as the sum of $n$ independent Erlang distributions, for $n \in \mathbb{N}$. An Erlang distribution is defined by two parameters, 
a number of i.i.d. exponential stages and a rate per stage. Thus, the general hypoexponential distribution is completely defined by the couples of parameters $\left(\lambda_{i}, k_{i}\right)$ for $i=1, \ldots, n$. Each couple $\left(\lambda_{i}, k_{i}\right)$ defines an Erlang distribution $\left(\lambda_{i} \in \mathbb{R}, k_{i} \in \mathbb{N}\right)$, and the rates $\lambda_{i}$ for $i=1, \ldots, n$ are all distinct. We denote by $K_{i}=k_{1}+k_{2}+\ldots+k_{i}$ for $i=1, \ldots, n$ and use the convention $K_{0}=k_{0}=0$. The cumulative distribution function (cdf) of the hypoexponential distribution is then given by

$$
F(x)=1-\boldsymbol{\alpha} e^{x M} \mathbf{1}
$$

for $x \geq 0$, where 1 is a column vector of size $K_{n}$ with ones everywhere, $\boldsymbol{\alpha}$ is a line vector of size $K_{n}$ and is given by $\boldsymbol{\alpha}=(1,0, \ldots, 0)$, and $e^{(.)}$denotes the exponential operator. The generator square matrix $M$ of size $K_{n} \times K_{n}$ is defined by the coefficients $m_{i, j}$ for $i, j \in\left\{1, \ldots, K_{n}\right\}$. We have $m_{j, j}=-\lambda_{i}$ and $m_{j, j+1}=\lambda_{i}$, for $K_{i-1}+1 \leq j \leq K_{i}$ and $i=1, \ldots, n$. All remaining coefficients of $M$ are zero. We thus may write

$$
M=\left(\begin{array}{ccccccccc}
-\lambda_{1} & \lambda_{1} & 0 & \ldots & 0 & 0 & 0 & \ldots & 0 \\
0 & -\lambda_{1} & \lambda_{1} & \ddots & 0 & 0 & 0 & \ldots & 0 \\
\vdots & \ddots & \ddots & \ddots & \ddots & \vdots & \vdots & \ddots & 0 \\
0 & 0 & \ldots & 0 & -\lambda_{1} & \lambda_{1} & 0 & \ldots & 0 \\
0 & 0 & \ldots & 0 & 0 & -\lambda_{2} & \lambda_{2} & \ldots & 0 \\
\vdots & \vdots & \vdots & \vdots & \vdots & \vdots & \vdots & \ddots & \vdots \\
0 & \ldots & \ldots & \ldots & \ldots & \ldots & 0 & -\lambda_{n} & \lambda_{n} \\
0 & \ldots & \ldots & \ldots & \ldots & \ldots & \ldots & \ldots & -\lambda_{n}
\end{array}\right) .
$$

Scheuer (1988) provides a formula for $F($.$) that involves high order derivatives of products of$ multiple functions. The formula is however hard to compute numerically. Amari and Misra (1997) propose a simplification of Scheuer (1988)'s formula using Laplace transforms and multi-function generalization of the Lebnitz rule for higher order derivatives of products of two functions. For a particular case with constraints on the values of the $\lambda_{i}$ s, Van Khuong and Kong (2006) provide the probability distribution function by inverting its Fourier transform. Using the Wilk's integral representation of the distribution of the product of independent beta random variables, Favaro and Walker (2010) provide an alternative formula for $F($.$) . We also refer the reader for more details to$ the review by Nadarajah (2008).

In this paper, we propose an alternative simple approach to analytically derive the cdf of $F($.$) . It$ 
is based on a linear algebraic matrix analysis. The structure of the approach is as follows. We first obtain some particular eigenvectors of the generator matrix $M$. These are next used to construct a new basis of vectors. The new basis allows to find the Jordan-Chevalley decomposition of $M$ into a sum of two commutative linear operators, a diagonal one and a nilpotent one. The exponential of the matrix $M$ then simply follows by inverting the new basis matrix using the Cayley-Hamilton theorem, which leads to the cdf of $F($.$) .$

\section{The Result}

Lemma 1 provides the eigenvalues of the matrix $M$, and one eigenvector associated to each eigenvalue.

Lemma 1 The eigenvalues of $M$ are $-\lambda_{i}$ for $i=1, \ldots, n$. An eigenvector of size $K_{n}$ associated to $-\lambda_{i}$ is the column vector $u_{i}$, where the coefficients of $u_{i}$, denoted by $u_{i, l}$ for $1 \leq l \leq K_{n}$, are given by

$$
\begin{cases}u_{i, l}=1 & , l=K_{i-1}+1, \\ u_{i, l}=0 & , l>K_{i-1}+1, \\ u_{i, l}=\left(\frac{\lambda_{i-1}}{\lambda_{i-1}-\lambda_{i}}\right)^{K_{i-1}-l+1} & , K_{i-2}+1 \leq l \leq K_{i-1}, \\ u_{i, l}=\prod_{j=1}^{i-(m+1)}\left(\frac{\lambda_{i-j}}{\lambda_{i-j}-\lambda_{i}}\right)^{k_{i-j}}\left(\frac{\lambda_{m}}{\lambda_{m}-\lambda_{i}}\right)^{K_{m}-l+1} & , K_{m-1}+1 \leq l \leq K_{m} \text { and } 0 \leq m<i-1 .\end{cases}
$$

Proof. Since $M$ is a triangular matrix, its eigenvalues are its diagonal coefficients, i.e., $-\lambda_{i}$ for $i=0, \ldots, n$. For $0 \leq i=0, \ldots, n$, consider the column vector $u_{i}$ defined with its coefficients $u_{i, l}$ $\left(1 \leq l \leq K_{n}\right)$, where $u_{i, K_{i-1}+1}=1, u_{i, l}=0$ for $l>K_{i-1}+1, u_{i, l}=\left(\frac{\lambda_{i-1}}{\lambda_{i-1}-\lambda_{i}}\right)^{K_{i-1}-l+1}$ for $K_{i-2}+1 \leq l \leq K_{i-1}$, and $u_{i, l}=\prod_{j=1}^{i-(m+1)}\left(\frac{\lambda_{i-j}}{\lambda_{i-j}-\lambda_{i}}\right)^{k_{i-j}}\left(\frac{\lambda_{m}}{\lambda_{m}-\lambda_{i}}\right)^{K_{m}-l+1}$, for $K_{m-1}+1 \leq l \leq K_{m}$ and $0 \leq m<i-1$. We now define, for $0 \leq i=0, \ldots, n, v_{i}$ as $v_{i}=M u_{i}$ and we denote its coefficients by $v_{i, l}$, for $1 \leq l \leq K_{n}$. Consider $1 \leq j \leq n$. For $K_{j-1}+1 \leq l \leq K_{j}$ and $l \neq K_{n}$, we have

$$
v_{i, l}=-\lambda_{j} u_{i, l}+\lambda_{j} u_{i, l+1}
$$

and $v_{i, K_{n}}=-\lambda_{n} u_{i, K_{n}}$. Since $u_{i, l}=0$ and $u_{i, K_{i-1}+1}=1$ for $l>K_{i-1}+1$, we deduce from Equation (3) that $v_{i, l}=0$ for $l>K_{i-1}+1$ and $v_{i, K_{i-1}+1}=-\lambda_{i}$. For $K_{i-2}+1 \leq l \leq K_{i-1}$ we 
have $u_{i, l}=\left(\frac{\lambda_{i-1}}{\lambda_{i-1}-\lambda_{i}}\right)^{K_{i-1}-l+1}$, and for $K_{m-1}+1 \leq l \leq K_{m}$ and $0 \leq m<i-1$ we have $u_{i, l}=$ $\prod_{j=1}^{i-(m+1)}\left(\frac{\lambda_{i-j}}{\lambda_{i-j}-\lambda_{i}}\right)^{k_{i-j}}\left(\frac{\lambda_{m}}{\lambda_{m}-\lambda_{i}}\right)^{K_{m}-l+1}$. Equation (3) therefore leads to $v_{i, l}=-\lambda_{i-1}\left(\frac{\lambda_{i-1}}{\lambda_{i-1}-\lambda_{i}}\right)^{K_{i-1}-l+1}+$ $\lambda_{i-1}\left(\frac{\lambda_{i-1}}{\lambda_{i-1}-\lambda_{i}}\right)^{K_{i-1}-(l+1)+1}=-\lambda_{i}\left(\frac{\lambda_{i-1}}{\lambda_{i-1}-\lambda_{i}}\right)^{K_{i-1}-l+1}$, for $K_{i-2}+1 \leq l \leq K_{i-1}$. We also obtain $v_{i, l}=-\lambda_{i} \prod_{j=1}^{i-(m+1)}\left(\frac{\lambda_{i-j}}{\lambda_{i-j}-\lambda_{i}}\right)^{k_{i-j}}\left(\frac{\lambda_{m}}{\lambda_{m}-\lambda_{i}}\right)^{K_{m}-l+1}$, for $K_{m-1}+1 \leq l \leq K_{m}$ and $0 \leq m<i-1$. This proves that $u$ is an eigenvector associated to the eigenvalue $-\lambda_{i}$, for $i=1, \ldots, n$, and finishes the proof of the lemma.

Let us denote by $\mathcal{B}$ the standard basis composed by the family of column vectors $e_{l}$, for $1 \leq l \leq$ $K_{n}$. The coefficients of $e_{l}\left(1 \leq l \leq K_{n}\right)$ are all zero except the coefficient in line $l$ which is equal to one. Consider now a new family of vectors denoted by $\mathcal{B}^{\prime}$ and composed by the vectors $e_{l}^{\prime}$ for $1 \leq l \leq K_{n}$, where $e_{K_{i-1}+1}^{\prime}=u_{i}$, and $e_{l}^{\prime}=e_{l}$ for $l \neq K_{i-1}+1$ and $i=1, \ldots n$. In Theorem 1 , we prove that $\mathcal{B}^{\prime}$ is a basis.

Theorem 1 The family of vectors $B^{\prime}$ is a basis.

Proof. The number of vectors in $\mathcal{B}^{\prime}$ is equal to the space dimension, $K_{n}$. It thus remains to prove that the family of vectors $\mathcal{B}^{\prime}$ is linearly independent. The proof is done by contradiction. Suppose that the family $\mathcal{B}^{\prime}$ is linearly dependent. Therefore, there exists some values $a_{1}, a_{2}, \ldots, a_{K_{n}} \in \mathbb{R}$, non all zero, such that $a_{1} e_{1}^{\prime}+a_{2} e_{2}^{\prime}+\cdots a_{K_{n}} e_{K_{n}}^{\prime}=0$. We have $e_{1}^{\prime}=u_{1}=e_{1}, e_{K_{1}+1}^{\prime}=u_{2}=$ $b_{2,1} e_{1}+b_{2,2} e_{2}+\cdots+b_{2, K_{1}+1} e_{K_{1}+1}, \cdots, e_{K_{n-1}+1}^{\prime}=u_{n}=b_{n, 1} e_{1}+b_{n, 2} e_{2}+\cdots+b_{n, K_{n-1}+1} e_{K_{n-1}+1}$, where the coefficients $b_{s, t}\left(1 \leq s \leq t \leq K_{n-1}+1\right)$, given by Lemma 1 , are all strictly positive. Then

$$
\begin{aligned}
& a_{1} e_{1}^{\prime}+a_{2} e_{2}^{\prime}+\cdots a_{K_{n}} e_{K_{n}}^{\prime} \\
& =a_{1}\left(1+b_{2,1}+b_{3,1}+\cdots b_{n, 1}\right) e_{1}+a_{2}\left(1+b_{2,2}+b_{3,2}+\cdots+b_{n, 2}\right) \\
& \quad+\cdots+a_{K_{n-1}+1} b_{n, K_{n-1}+1} e_{K_{n-1}+1}+a_{K_{n-1}+2} e_{K_{n-1}+2}+\cdots+a_{K_{n}} e_{K_{n}} \\
& \quad+\cdots \\
& =0
\end{aligned}
$$

Since the coefficients $b_{s, t}\left(1 \leq s \leq t \leq K_{n-1}+1\right)$ are all strictly positive and $\left(e_{1}, e_{2}, \ldots, e_{K_{n}}\right)$ is the Basis $\mathcal{B}$, we should have $a_{1}=a_{2}=\ldots=a_{K_{n}}=0$, which is absurd. Thus $\mathcal{B}^{\prime}$ is a basis.

Let us now proceed to a change of basis from $\mathcal{B}$ to $\mathcal{B}^{\prime}$. We want to write $M$ in the new basis $\mathcal{B}^{\prime}$, which leads to a matrix denoted by $M^{\prime}$. We denote by $P$ the new basis matrix allowing to move 
from $\mathcal{B}$ to basis $\mathcal{B}^{\prime}$. This means that $P$ is given by the vectors of the old basis $\mathcal{B}$ but written in the new basis $\mathcal{B}^{\prime}$. We have $M=P M^{\prime} P^{-1}$. We next give $P$ and $P^{\prime}$. We have

$$
P=\left(\begin{array}{ccccc}
I_{k_{1}} & P_{1,2} & P_{1,3} & \ldots & P_{1, n} \\
O_{2,1} & I_{k_{2}} & P_{2,3} & \ldots & P_{2, n} \\
\vdots & \ddots & \ddots & \ddots & \vdots \\
\vdots & \ddots & \ddots & \ddots & P_{n-1, n} \\
O_{n, 1} & \ldots & \ldots & O_{n, n-1} & I_{k_{n}}
\end{array}\right)
$$

where $P_{i, j}$, for $i, j=1, \cdots, n$ and $i<j$, is a matrix of size $k_{i} \times k_{j}$ with 0 everywhere except in the first column. The coefficient of line $k\left(1 \leq k \leq k_{i}\right)$ of the first column of $P_{i, j}$ is

$$
\prod_{l=i+1}^{j-1}\left(\frac{\lambda_{l}}{\lambda_{l}-\lambda_{j}}\right)^{k_{l}}\left(\frac{\lambda_{i}}{\lambda_{i}-\lambda_{j}}\right)^{k_{i}-k+1} .
$$

The matrix $I_{k_{i}}$, for $i=1, \ldots, n$, is the identity matrix of size $k_{i} \times k_{i}$, and the matrix $O_{i, j}$, for $i, j=1, \cdots, n$, of size $k_{i} \times k_{j}$ has zero coefficients everywhere. We now compute $P^{-1}$ using the Cayley-Hamilton theorem (see for example Gourdon (1994)). Since $\left(I_{K_{n}}-P\right)^{n}=O_{K_{n}}$, we deduce using the binomial theorem that $\sum_{k=0}^{n}\left(\begin{array}{l}n \\ k\end{array}\right)(-1)^{k} P^{k}=O_{K_{n}}$, where $\left(\begin{array}{l}n \\ k\end{array}\right)=\frac{n !}{k !(n-k) !}$ for $n \geq 0$ and $0 \leq$ $k \leq n$. Thus $I_{K_{n}}=-\sum_{k=1}^{n}\left(\begin{array}{l}n \\ k\end{array}\right)(-1)^{k} P^{k}=P\left(-\sum_{k=1}^{n}\left(\begin{array}{l}n \\ k\end{array}\right)(-1)^{k} P^{k-1}\right)$. The matrix $-\sum_{k=1}^{n}\left(\begin{array}{l}n \\ k\end{array}\right)(-1)^{k} P^{k-1}$ commutes with $P$. We therefore obtain $P^{-1}=-\sum_{k=1}^{n}\left(\begin{array}{l}n \\ k\end{array}\right)(-1)^{k} P^{k-1}$.

In the basis $\mathcal{B}^{\prime}$, the generator matrix is

$$
M^{\prime}=\left(\begin{array}{ccccc}
E_{1} & O_{1,2} & O_{1,3} & \ldots & O_{1, n} \\
O_{2,1} & E_{2} & O_{2,3} & \ldots & O_{2, n} \\
\vdots & \ddots & \ddots & \ddots & \vdots \\
\vdots & \ddots & \ddots & \ddots & O_{n-1, n} \\
O_{n, 1} & \ldots & \ldots & O_{n, n-1} & E_{n}
\end{array}\right)
$$

where $E_{i}(i=1, \cdots, n)$ is a sub-matrix of size $k_{i} \times k_{i}$. It is given by

$$
E_{i}=\left(\begin{array}{cccccc}
-\lambda_{i} & \lambda_{i} & 0 & \ldots & 0 & 0 \\
0 & -\lambda_{i} & \lambda_{i} & \ddots & 0 & 0 \\
\vdots & \ddots & \ddots & \ddots & \ddots & \vdots \\
0 & 0 & \ddots & -\lambda_{i} & \lambda_{i} & 0 \\
0 & 0 & \ldots & 0 & -\lambda_{i} & \lambda_{i} \\
0 & 0 & \ldots & 0 & 0 & -\lambda_{i}
\end{array}\right)
$$


For $i=1, \cdots, n, E_{i}$ may be written as $E_{i}=D_{i}+N_{i}$ with

$$
D_{i}=\left(\begin{array}{cccccc}
-\lambda_{i} & 0 & 0 & \ldots & 0 & 0 \\
0 & -\lambda_{i} & 0 & \ddots & 0 & 0 \\
\vdots & \ddots & \ddots & \ddots & \ddots & \vdots \\
0 & 0 & \ddots & -\lambda_{i} & 0 & 0 \\
0 & 0 & \ldots & 0 & -\lambda_{i} & 0 \\
0 & 0 & \ldots & 0 & 0 & -\lambda_{i}
\end{array}\right) \text {, and } N_{i}=\left(\begin{array}{cccccc}
0 & \lambda_{i} & 0 & \ldots & 0 & 0 \\
0 & 0 & \lambda_{i} & \ddots & 0 & 0 \\
\vdots & \ddots & \ddots & \ddots & \ddots & \vdots \\
0 & 0 & \ddots & 0 & \lambda_{i} & 0 \\
0 & 0 & \ldots & 0 & 0 & \lambda_{i} \\
0 & 0 & \ldots & 0 & 0 & 0
\end{array}\right) .
$$

Using the sub-matrices $D_{i}$ and $N_{i}$, we define the matrices $D$ and $N$ as

$$
D=\left(\begin{array}{ccccc}
D_{1} & O_{1,2} & O_{1,3} & \ldots & O_{1, n} \\
O_{2,1} & D_{2} & O_{2,3} & \ldots & O_{2, n} \\
\vdots & \ddots & \ddots & \ddots & \vdots \\
\vdots & \ddots & \ddots & \ddots & O_{n-1, n} \\
O_{n, 1} & \ldots & \ldots & O_{n, n-1} & D_{n}
\end{array}\right) \text {, and } N=\left(\begin{array}{ccccc}
N_{1} & O_{1,2} & O_{1,3} & \ldots & O_{1, n} \\
O_{2,1} & N_{2} & O_{2,3} & \ldots & O_{2, n} \\
\vdots & \ddots & \ddots & \ddots & \vdots \\
\vdots & \ddots & \ddots & \ddots & O_{n-1, n} \\
O_{n, 1} & \ldots & \ldots & O_{n, n-1} & N_{n}
\end{array}\right) .
$$

Therefore $M^{\prime}=D+N$. Since $N^{\max \left(k_{1}, k_{2}, \cdots, k_{n}\right)}=O_{K_{n}, K_{n}}$, the matrix $N$ is nilpotent. Note that $D$ is a diagonal matrix. Then, $D$ commutes with any other matrix, in particular $N$. Therefore the decomposition of $M^{\prime}$ into $M^{\prime}=N+D$ is the unique Jordan-Chevalley decomposition of $M^{\prime}$ into a summation of two commutative matrices, a nilpotent one and a diagonalisable one. Using the new basis matrix $P$, we have $M=P M^{\prime} P^{-1}=P(N+D) P^{-1}$, so, $e^{x M}=P e^{x(N+D)} P^{-1}$ (see Gourdon (1994)). Since $N$ and $D$ commute, we deduce that $e^{x(N+D)}=e^{x D} \times e^{x N}$. Because $x D$ is a diagonal matrix we have

$$
e^{x D}=\left(\begin{array}{ccccc}
e^{x D_{1}} & O_{1,2} & O_{1,3} & \ldots & O_{1, n} \\
O_{2,1} & e^{x D_{2}} & O_{2,3} & \ldots & O_{2, n} \\
\vdots & \ddots & \ddots & \ddots & \vdots \\
\vdots & \ddots & \ddots & \ddots & O_{n-1, n} \\
O_{n, 1} & \ldots & \ldots & O_{n, n-1} & e^{x D_{n}}
\end{array}\right)
$$

with

$$
e^{x D_{i}}=\left(\begin{array}{cccc}
e^{-x \lambda_{i}} & 0 & \cdots & 0 \\
0 & e^{-x \lambda_{i}} & \ddots & 0 \\
\vdots & \ddots & \ddots & \vdots \\
0 & 0 & \cdots & e^{-x \lambda_{i}}
\end{array}\right)
$$


for $i=1, \cdots, n$. Recall that $e^{x N}$ is given by an infinite summation, $e^{x N}=\sum_{j=0}^{+\infty} \frac{(x N)^{j}}{j !}$. However, since $N$ is nilpotent (for $i=1, \cdots, n$, we have $N_{i}^{k_{i}}=O_{k_{i}, k_{i}}$ ), the evaluation of $\sum_{j=0}^{+\infty} \frac{(x N)^{j}}{j !}$ is done within a finite number of calculations. We have $e^{x N}=\sum_{j=0}^{\max \left(k_{1}, k_{2}, \cdots, k_{n}\right)-1} \frac{(x N)^{j}}{j !}$. Then,

$$
N^{j}=\left(\begin{array}{ccccc}
N_{1}^{j} & O_{1,2} & O_{1,3} & \ldots & O_{1, n} \\
O_{2,1} & N_{2}^{j} & O_{2,3} & \ldots & O_{2, n} \\
\vdots & \ddots & \ddots & \ddots & \vdots \\
\vdots & \ddots & \ddots & \ddots & O_{n-1, n} \\
O_{n, 1} & \ldots & \ldots & O_{n, n-1} & N_{n}^{j}
\end{array}\right)
$$

for $j \in \mathbb{N}$, where the matrix $N_{i}^{j}$ is of size $k_{i} \times k_{i}(i=1, \cdots, n)$. The $N_{i}^{j}$ 's coefficients of line $s$ and column $t$ are all zero except if $t-s=j$ and $j=0, \cdots k_{i}$ where the coefficients are all equal to $\lambda_{i}^{j}$. As a consequence

$$
e^{x N}=\left(\begin{array}{ccccc}
e^{x N_{1}} & O_{1,2} & O_{1,3} & \ldots & O_{1, n} \\
O_{2,1} & e^{x N_{2}} & O_{2,3} & \ldots & O_{2, n} \\
\vdots & \ddots & \ddots & \ddots & \vdots \\
\vdots & \ddots & \ddots & \ddots & O_{n-1, n} \\
O_{n, 1} & \ldots & \ldots & O_{n, n-1} & e^{x N_{n}}
\end{array}\right)
$$

where

$$
e^{x N_{i}}=\left(\begin{array}{cccccc}
1 & x \lambda_{i} & \frac{x^{2}}{2} \lambda_{i}^{2} & \ldots & \frac{x^{k_{i}-2}}{\left(k_{i}-2\right) !} \lambda_{i}^{k_{i}-2} & \frac{x^{k_{i}-1}}{\left(k_{i}-1\right) !} \lambda_{i}^{k_{i}-1} \\
0 & 1 & x \lambda_{i} & \ddots & \frac{x^{k_{i}-3}}{\left(k_{i}-3\right) !} \lambda_{i}^{k_{i}-3} & \frac{x^{k_{i}-2}}{\left(k_{i}-2\right) !} \lambda_{i}^{k_{i}-2} \\
\vdots & \ddots & \ddots & \ddots & \ddots & \vdots \\
0 & 0 & \ddots & 1 & x \lambda_{i} & \frac{x^{2}}{2} \lambda_{i}^{2} \\
0 & 0 & \ldots & 0 & 1 & x \lambda_{i} \\
0 & 0 & \ldots & 0 & 0 & 1
\end{array}\right),
$$

for $i=1, \cdots, n$. Finally, we may write

$$
e^{x(N+D)}=\left(\begin{array}{ccccc}
e^{x N_{1}} e^{x D_{1}} & O_{1,2} & O_{1,3} & \ldots & O_{1, n} \\
O_{2,1} & e^{x N_{2}} e^{x D_{2}} & O_{2,3} & \ldots & O_{2, n} \\
\vdots & \ddots & \ddots & \ddots & \vdots \\
\vdots & \ddots & \ddots & \ddots & O_{n-1, n} \\
O_{n, 1} & \ldots & \ldots & O_{n, n-1} & e^{x N_{n}} e^{x D_{n}}
\end{array}\right),
$$


where

$$
e^{x N_{i}} e^{x D_{i}}=\left(\begin{array}{cccccc}
e^{-x \lambda_{i}} & e^{-x \lambda_{i}} x \lambda_{i} & e^{-x \lambda_{i}} \frac{x^{2}}{2} \lambda_{i}^{2} & \ldots & e^{-x \lambda_{i}} \frac{x^{k_{i}-2}}{\left(k_{i}-2\right) !} \lambda_{i}^{k_{i}-2} & e^{-x \lambda_{i}} \frac{x^{k_{i}-1}}{\left(k_{i}-1\right) !} \lambda_{i}^{k_{i}-1} \\
0 & e^{-x \lambda_{i}} & e^{-x \lambda_{i}} x \lambda_{i} & \ddots & e^{-x \lambda_{i}} \frac{x^{k_{i}-3}}{\left(k_{i}-3\right) !} \lambda_{i}^{k_{i}-3} & e^{-x \lambda_{i}} \frac{x^{k_{i}-2}}{\left(k_{i}-2\right) !} \lambda_{i}^{k_{i}-2} \\
\vdots & \ddots & \ddots & \ddots & \ddots & \vdots \\
0 & 0 & \ddots & e^{-x \lambda_{i}} & e^{-x \lambda_{i}} x \lambda_{i} & e^{-x \lambda_{i} \frac{x^{2}}{2} \lambda_{i}^{2}} \\
0 & 0 & \ldots & 0 & e^{-x \lambda_{i}} & e^{-x \lambda_{i}} x \lambda_{i} \\
0 & 0 & \ldots & 0 & 0 & e^{-x \lambda_{i}}
\end{array}\right),
$$

for $i=1, \cdots, n$.

In order to obtain the cdf of the hypoexponential distribution we only need to evaluate the sum of the coefficients of the first line of the matrix $e^{x M}$. Note that in the particular case of an Erlang distribution $\left(k_{2}=k_{3}=\cdots=k_{n}=0\right)$ we have $P=P^{-1}=I_{k_{1}}$. We then easily obtain the well-known expression $F(x)=1-e^{-x \lambda_{1}} \sum_{k=0}^{k=k_{1}-1} \frac{\left(\lambda_{1} x\right)^{k}}{k !}$, for $x \geq 0$, by summing the coefficients of the first line of $e^{x N_{1}} e^{x D_{1}}$

We have

$$
P e^{x(D+N)}=\left(\begin{array}{ccccc}
e^{x\left(N_{1}+D_{1}\right)} & P_{1,2} e^{x\left(N_{2}+D_{2}\right)} & P_{1,3} e^{x\left(N_{3}+D_{3}\right)} & \ldots & P_{1, n} e^{x\left(N_{n}+D_{n}\right)} \\
O_{2,1} & e^{x\left(N_{2}+D_{2}\right)} & P_{2,3} e^{x\left(N_{3}+D_{3}\right)} & \ldots & P_{2, n} e^{x\left(N_{n}+D_{n}\right)} \\
\vdots & \ddots & \ddots & \ddots & \vdots \\
\vdots & \ddots & \ddots & \ddots & P_{n-1, n} e^{x\left(N_{n}+D_{n}\right)} \\
O_{n, 1} & \ldots & \ldots & O_{n, n-1} & e^{x\left(N_{n}+D_{n}\right)}
\end{array}\right),
$$

where

$$
\begin{aligned}
& P_{i, j} e^{x\left(N_{j}+D_{j}\right)}= \\
& e^{-x \lambda_{j}} \prod_{l=i+1}^{j-1}\left(\frac{\lambda_{l}}{\lambda_{l}-\lambda_{j}}\right)^{k_{l}}\left(\begin{array}{cccc}
\left(\frac{\lambda_{i}}{\lambda_{i}-\lambda_{j}}\right)^{k_{i}} & x \lambda_{j}\left(\frac{\lambda_{i}}{\lambda_{i}-\lambda_{j}}\right)^{k_{i}} & \ldots & \frac{x^{k_{j}-1}}{\left(k_{j}-1\right) !} \lambda_{j}^{k_{j}-1}\left(\frac{\lambda_{i}}{\lambda_{i}-\lambda_{j}}\right)^{k_{i}} \\
\left(\frac{\lambda_{i}}{\lambda_{i}-\lambda_{j}}\right)^{k_{i}-1} & x \lambda_{j}\left(\frac{\lambda_{i}}{\lambda_{i}-\lambda_{j}}\right)^{k_{i}-1} & \ldots & \frac{x^{k_{j}-1}}{\left(k_{j}-1\right) !} \lambda_{j}^{k_{j}-1}\left(\frac{\lambda_{i}}{\lambda_{i}-\lambda_{j}}\right)^{k_{i}-1} \\
\vdots & \ddots & \ddots & \vdots \\
\left(\frac{\lambda_{i}}{\lambda_{i}-\lambda_{j}}\right)^{2} & x \lambda_{j}\left(\frac{\lambda_{i}}{\lambda_{i}-\lambda_{j}}\right)^{2} & \ldots & \frac{x^{k_{j}-1}}{\left(k_{j}-1\right) !} \lambda_{j}^{k_{j}-1}\left(\frac{\lambda_{i}}{\lambda_{i}-\lambda_{j}}\right)^{2} \\
\left(\frac{\lambda_{i}}{\lambda_{i}-\lambda_{j}}\right)^{1} & x \lambda_{j}\left(\frac{\lambda_{i}}{\lambda_{i}-\lambda_{j}}\right)^{1} & \ldots & \frac{x^{k_{j}-1}}{\left(k_{j}-1\right) !} \lambda_{j}^{k_{j}-1}\left(\frac{\lambda_{i}}{\lambda_{i}-\lambda_{j}}\right)^{1}
\end{array}\right),
\end{aligned}
$$

for $i, j=1, \ldots n$ and $i<j$.

We then have to evaluate $P e^{x(D+N)} P^{-1}=\sum_{k=1}^{n}\left(\begin{array}{l}n \\ k\end{array}\right)(-1)^{k-1} P e^{x(D+N)} P^{k-1}$. Note again that $P$ can be written as $P=I_{K_{n}}+P-I_{K_{n}}$. The matrices $I_{K_{n}}$ and $P-I_{K_{n}}$ commute, $I_{K_{n}}$ is diagonal 
and $P-I_{K_{n}}$ is nilpotent. This implies

$$
\begin{aligned}
P e^{x(D+N)} P^{-1} & =\sum_{k=1}^{n}\left(\begin{array}{l}
n \\
k
\end{array}\right)(-1)^{k-1} P e^{x(D+N)}\left(I_{K_{n}}+P-I_{K_{n}}\right)^{k-1} \\
& =\sum_{k=1}^{n}\left(\begin{array}{l}
n \\
k
\end{array}\right)(-1)^{k-1} P e^{x(D+N)} \sum_{l=0}^{k-1}\left(\begin{array}{c}
k-1 \\
l
\end{array}\right)\left(P-I_{K_{n}}\right)^{l},
\end{aligned}
$$

or equivalently

$$
P e^{x(D+N)} P^{-1}=\sum_{k=1}^{n} \sum_{l=0}^{k-1}(-1)^{k-1}\left(\begin{array}{c}
n \\
k
\end{array}\right)\left(\begin{array}{c}
k-1 \\
l
\end{array}\right) P e^{x(D+N)}\left(P-I_{K_{n}}\right)^{l} .
$$

We have $\left(P-I_{K_{n}}\right)^{0}=I_{K_{n}}$, and for $1 \leq l \leq n-1$

$$
\left(P-I_{K_{n}}\right)^{l}=\left(\begin{array}{ccccc}
O_{1,1} & A_{1,2} & A_{1,3} & \ldots & A_{1, n} \\
O_{2,1} & O_{2,2} & A_{2,3} & \ldots & A_{2, n} \\
\vdots & \ddots & \ddots & \ddots & \vdots \\
\vdots & \ddots & \ddots & \ddots & A_{n-1, n} \\
O_{n, 1} & \ldots & \ldots & O_{n, n-1} & O_{n, n}
\end{array}\right),
$$

where $A_{i, j}=O_{i, j}$ for $j-i<l, i, j=1, \ldots, n$, otherwise $A_{i, j}=\sum_{i<a_{1}<a_{2}<\cdots<a_{l-1}<j} P_{i, a_{1}} P_{a_{1}, a_{2}} \cdots P_{a_{l-2}, a_{l-1}} P_{a_{l-1}, j}$. The matrix $P_{i, a_{1}} P_{a_{1}, a_{2}} \cdots P_{a_{l-2}, a_{l-1}} P_{a_{l-1}, j}$ is a matrix of dimension $k_{i} \times k_{j}$ where all coefficients are zero except the ones on the first column. The coefficient of line $k$ (for $1 \leq k \leq k_{i}$ ) of the first column is equal to

$$
\left(\frac{\lambda_{i}}{\lambda_{i}-\lambda_{a_{1}}}\right)^{k_{i}-k+1} \prod_{m=i+1}^{a_{1}-1}\left(\frac{\lambda_{m}}{\lambda_{m}-\lambda_{a_{1}}}\right)^{k_{m}} \prod_{n=a_{1}}^{a_{2}-1}\left(\frac{\lambda_{n}}{\lambda_{n}-\lambda_{a_{2}}}\right)^{k_{n}} \cdots \prod_{r=a_{l-1}}^{j-1}\left(\frac{\lambda_{r}}{\lambda_{r}-\lambda_{a_{j}}}\right)^{k_{r}} .
$$

We are only interested in the sum of the coefficients of the first line of $e^{x M}$. We thus evaluate the terms in the first line of $P e^{x(D+N)}\left(P-I_{K_{n}}\right)^{l}$, for $0 \leq l \leq n-1$. For $l=0, P e^{x(D+N)}\left(P-I_{K_{n}}\right)^{0}=$ $P e^{x(D+N)}$. Then, using Equation (18) we compute the terms in the first line of $P e^{x(D+N)}$. For $1 \leq l \leq n-1$, we have

$$
P e^{x(D+N)}\left(P-I_{K_{n}}\right)^{l}=\left(\begin{array}{ccccc}
O_{1,1} & B_{1,2} & B_{1,3} & \ldots & B_{1, n} \\
O_{2,1} & O_{2,2} & B_{2,3} & \ldots & B_{2, n} \\
\vdots & \ddots & \ddots & \ddots & \vdots \\
\vdots & \ddots & \ddots & \ddots & B_{n-1, n} \\
O_{n, 1} & \ldots & \ldots & O_{n, n-1} & O_{n, n}
\end{array}\right),
$$

where $B_{i, j}=O_{i, j}$ for $j-i<l, i, j=1, \ldots, n$, otherwise for $i=1$ and $1<j<n, B_{1, j}=$ $\sum_{s=1}^{j-1} P_{1, s} e^{x\left(N_{s}+D_{s}\right)} \sum_{s<a_{1}<a_{2}<\cdots<a_{l-1}<j} P_{s, a_{1}} P_{a_{1}, a_{2}} \cdots P_{a_{l-2}, a_{l-1}} P_{a_{l-1}, j}$ with the convention that $P_{1,1}=$ $I_{k_{1}}$. Therefore, the cdf of the hypoexponential distribution is given by 


$$
\begin{aligned}
& F(x)=1- \\
& \sum_{k=1}^{n} \sum_{l=0}^{k-1}(-1)^{k-1}\left(\begin{array}{c}
n \\
k
\end{array}\right)\left(\begin{array}{c}
k-1 \\
l
\end{array}\right) \sum_{j=1}^{n} \sum_{s=1}^{j-1} e^{-x \lambda_{s}} \prod_{l=1}^{s-1}\left(\frac{\lambda_{l}}{\lambda_{l}-\lambda_{s}}\right)^{k_{s}} \\
& \quad \times \sum_{s<a_{1}<\cdots<a_{l-1}<j}\left(\frac{\lambda_{s}}{\lambda_{s}-\lambda_{a_{1}}}\right)^{k_{s}} \prod_{m=s+1}^{a_{1}-1}\left(\frac{\lambda_{m}}{\lambda_{m}-\lambda_{a_{1}}}\right)^{k_{m}} \prod_{n=a_{1}}^{a_{2}-1}\left(\frac{\lambda_{n}}{\lambda_{n}-\lambda_{a_{2}}}\right)^{k_{n}} \\
& \cdots \prod_{r=a_{l-1}}^{j-1}\left(\frac{\lambda_{r}}{\lambda_{r}-\lambda_{a_{j}}}\right)^{k_{r}} \sum_{q=0}^{k_{s}-1} \frac{\left(\left(\lambda_{s}-\lambda_{a_{1}}\right) x\right)^{q}}{q !},
\end{aligned}
$$

for $x \geq 0$.

\section{Conclusion}

This paper proposes a new approach based on linear algebraic matrix analysis for the computation of the cumulative distribution function of the sum of independent Erlang random variables. This is a simpler alternative approach to the existing ones in the literature. However, direct computation of the involved expressions may lead to numerical difficulties. As a future research, it would be interesting to develop an efficient numerical method to avoid numerical round of errors.

\section{Acknowledgements}

This work was supported by Agence Nationale de la Recherche under the project ANR-JCJCSIMI3-2012-OPERA. We also want to express our gratitude to the anonymous reviewers and the associate editor for their useful comments, that significantly improved this paper.

\section{References}

Amari, S. and Misra, R. (1997). Closed-form expressions for distribution of sum of exponential random variables. IEEE Transactions on Reliability, 46:519-522.

Favaro, S. and Walker, S. (2010). On the distribution of sums of independent exponential random variables via wilks' integral representation. Acta applicandae mathematicae, 109(3):1035-1042. 
Gourdon, X. (1994). Les maths en tête : Algèbre. Ellipses, Paris.

Nadarajah, S. (2008). A review of results on sums of random variables. Acta Applicandae Mathematicae, 103(2):131-140.

Scheuer, E. (1988). Reliability of an m-out of-n system when component failure induces higher failure rates in survivors. IEEE Transactions on Reliability, 37(1):73-74.

Van Khuong, H. and Kong, H. (2006). General expression for pdf of a sum of independent exponential random variables. IEEE Communications Letters, 10(3):159-161. 